\title{
Space-Charge-Dominated Mass Spectrometry Ion Sources: Modeling and Sensitivity
}

\author{
Mark Busman and Jan Sunner \\ Department of Chemistry \\ Curtis R. Vogel \\ Department of Mathematical Sciences, Montana State University, Bozeman, Montana, USA
}

\begin{abstract}
The factors determining the sensitivity of space-charge-dominated (SCD) unipolar ion sources, such as electrospray (ESP) and corona atmospheric pressure ionization (API) have been studied theoretically. The most important parameters are the ion density and ion drift time in the vicinity of the sampling orifice. These are obtained by solving a system of differential equations, "the space-charge problem." For some simple geometries, analytical solutions are known. For a more realistic "needle-in-can" geometry, a solution to the space-charge problem was obtained using a finite-element method. The results illustrate some general characteristics of SCD ion sources. It is shown that for typical operating conditions the minimum voltage required to overcome the space-charge effect in corona API or ESP ion sources constitutes a dominant or significant fraction of total applied voltage. Further, the electric field and the ion density in the region of the ion-sampling orifice as well as the ion residence time in the source are determined mainly by the space charge. Finally, absolute sensitivities of corona API ion sources were calculated by using a geometry-independent treatment of space charge. (J Am Soc Mass Spectrom 1991, 2, 1-10)
\end{abstract}

$\mathrm{M}$ ass spectrometric techniques using highpressure ion sources are becoming increasingly important in analytical chemistry. Atmospheric pressure ionization mass spectrometry (API/MS) is used for analysis of sub-parts per billion levels of trace gases in ambient air [1-4]. Equipped with a nebulizer, such a snurce becomes the interface in a liquid chromatograph/mass spectrometer [5]. In these methods, the atmospheric gas is usually ionized by a corona discharge, but ${ }^{63} \mathrm{Ni}$ is also used. In electrospray (ESP) $[6,7]$, the ions instead originate from a charged spray. The electron capture detector (ECD) also operates at atmospheric pressure [8-10]. In ion mobility spectrometry (IMS), ions are separated on the basis of their mobility in atmospheric gas $[11,12]$.

In the API devices mentioned above, except the ion mobility spectrometer and the ECD, the ions pass through a sampling orifice into the vacuum system for detection by mass spectrometry. The analytical signal thus depends on the efficiency of analyte ion formation (and destruction), of analyte ion transport to the vicinity of the orifice, of analyte ion extraction through

Address reprint requests to Jan Sunner, Department of Chemistry, Montana State University, Bozeman, MT 59717. the orifice, and of ion extraction from the supersonic jet, as well as on the transmission through the mass spectrometer. We will discuss the processes up to the orifice.

For the understanding of ion formation and transport it is useful to categorize ion sources with respect to the major ion loss mechanism. At low in densitiies, ions are lost mainly by diffusion (diffusiondominated loss) or by drift in the Laplacian field, that is, the electric field created by applying different potentials to the walls or electrodes in the ion source (Laplacian field dominated loss). At increasing ion densities in the bipolar (ions of both polarities present) ion source, diffusion first becomes ambipolar [13]) and is then replaced by ion-ion (or ion-electron) recombination as the main ion loss mechanism (recumbination-dominated loss). This is the situation when ${ }^{63} \mathrm{Ni}$ causes the ionization, as in the ECD [14] and in some API [15] and IMS sources [11]. Models of recombination-dominated ion sources have been published [16, 17]. The unipolar ion source (ions of only one polarity present) becomes space-charge-dominated (SCD) at high ion density. The electric feld within the source is then determined mainly by the space charge, and ion drift to the walls is the dominating ion loss mechanism. 
This is the case, for example, in the ECD directly after the electron-extraction pulse has been applied to the anode [14].

Of the different types of ion sources, the SCD one is the least understood. For ESP, corona API, and IMS, the problem is to what extent ion transport is determined by the space charge as opposed to the kilovolt potential applied to the various electrodes. In mass spectrometry, the ECD model of Gobby et al. [14] is perhaps the only quantitative treatment of space-charge effects at high pressure. However, results in discharge physics [18] are often applicable to mass spectrometry ion sources. Thus, space-charge effects in systems with simple geometries were treated by Townsend [19]. A related low-pressure treatment is due to Langmuir [20]. A discussion of a spherical system can be found, for example, in ref. 21. A model of a point-to-plane corona that considers space-charge effects has been presented by Sigmond [22]. However, the implications of these results for mass spectrometric ion sources, in particular with regard to sensitivity, seem never to have been investigated.

Space-charge effects are important not only in discharge physics but also for many devices in science and technology. Examples are aerosol precipitators [23], membrane ion exchangers [24], and semiconductor devices [25]. The modeling of these, as well as of space-charge effects in gases, is part of the subject of nonlinear transport theory [24].

The relationship between the charge density $\rho$ and the electrostatic potential $U$ is given by Gauss's law (using SI units),

$$
-\bar{\nabla} \cdot \bar{\nabla} U=\bar{\nabla} \cdot \tilde{E}=\rho / \epsilon
$$

where $\bar{E}$ is the electric field vector and $\epsilon$ is the electric permittivity. Assuming no ionization, the continuity equation is

$$
\frac{\partial \rho}{\partial t}+\bar{\nabla} \cdot \bar{J}=0
$$

where $j$ is the ion current density vector. Here we are interested in steady-state solutions, and eq 2 simplifies to

$$
\bar{\nabla} \cdot \bar{J}=0
$$

At high pressure, the main ion transport processes are ion drift, diffusion, and convection. The ion current density is given by

$$
\bar{J}=\kappa \rho \bar{E}-D \bar{\nabla} \rho+\rho \bar{F}
$$

where $\kappa$ is the coefficient of ion mobility, $D$ is the diffusion constant, and $\bar{F}$ is the gas flow vector. In a typical SCD ion source, diffusion is important only very close to the walls, and convection mainly affects the efficiency of ion sampling. Thus, eq 4 simplifies to

$$
\bar{I}=\kappa \rho \bar{E}=-\kappa \rho \bar{\nabla} U
$$

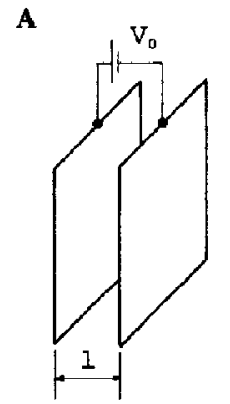

$\mathbf{B}$
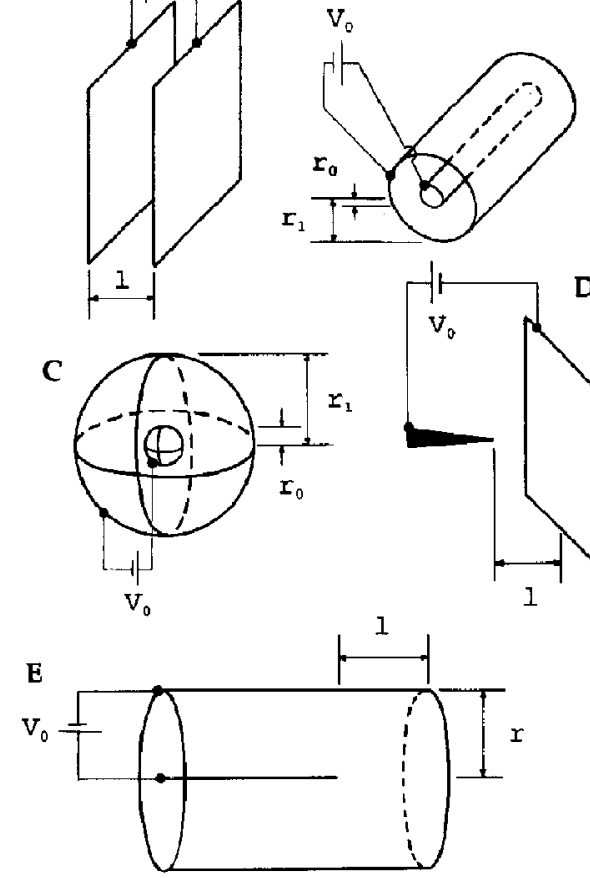

Figure 1. Simple geometries for SCD ion sources discussed in the text. (a) Planar geometry, infinite parallel planes; (b) cylindrical geometry, concentric cylinders of infinite length; (c) spherical geometry, concentric spheres; (d) point-to-plane geometry; (e) "needle-in-can" geometry. The ion supply surfaces are given a positive polarity in the figures.

Equations 1, 3, and 5 form a nonlinear system of partial differential equations, the "space-charge problem." The boundary conditions may be specified by determining some combination of the component of $E$ normal to the surface or $U$ on the surface, $\delta \Omega$, of a region $\Omega$, for example,

$$
U=U_{\text {bndry }}(x), \quad x \in \delta \Omega
$$

and similarly for $\nabla \rho$ or $\rho$. The solution to these equations should give the electric field distribution throughout the ion source as well as the potential distribution, the ion density distribution, and ion drift times.

Neglecting diffusion, the space-charge problem, eqs 1,3 , and 5 , has analytical solutions for some simple geometries: (1) infinite parallel plates, (2) two concentric cylinders, and (3) two concentric spheres; see Figure $1[19,21,26]$. The electric field between two parallel plates (Figure 1a), is given by

$$
E(x)=\left(\frac{2 J}{\kappa \epsilon} x+E_{0}^{2}\right)^{1 / 2}
$$

where $J$ is the ion current density, $k$ is the ion 
mobility, $E_{0}$ is the electric field at the "current source" plate or "ion supply surface," and $x$ is the distance from this surface.

In the spherical geometry, where the inner concentric sphere is the ion supply surface (Figure 1c), the electric field is given by

$$
E(r)=\left(\frac{r_{0}}{r}\right)^{2}\left(E_{0}^{2}-\frac{2 J_{0}}{3 \kappa \epsilon r_{0}^{2}}\left(r_{0}^{3}-r^{3}\right)\right)^{1 / 2}
$$

where $r$ is the radius, $r_{0}$ is the radius of the inner sphere, $E_{0}$ is the electric field, and $J_{0}$ is the ion current density at the inner sphere surface. $J_{0}$ is related to the discharge current $I_{0}$ by

$$
J_{0}=I_{0} /\left(4 \pi r_{0}^{2}\right)
$$

It is noteworthy that in both the planar and spherical cases, eqs 7 and 8 , the space-charge solution is a function of the boundary conditions at the ion supply surface only, that is, $E_{0}, r_{0}$, and $J_{0}$,

Equation 8 is easily integrated to give the potential distribution $U(r)$ and the overall drift voltage between the ion supply surface and the counter electrode, $V_{d}$. The charge density is obtained from eq 5 , and the ion drift time from the ion supply surface from

$$
t_{d}(r)=\int_{r=r_{0}}^{r} \frac{d r}{k E(r)}
$$

In previous articles, with Kebarle, we studied the factors that determine the sensitivities for analytes in ambient air corona API/MS [27, 28]. We were able to explain the relative sensitivities in terms of the thermodynamics and kinetics for protonation of the analytes by the main reagent ions, $\mathrm{H}_{3} \mathrm{O}^{+}\left(\mathrm{H}_{2} \mathrm{O}\right)_{h}$. However, we were not able to clarify what factors determine the absolute sensitivities in API. In the present article it is shown that the variation of absolute ion signals with important ion source parameters can be explained as a consequence of the ion source being space-charge-dominated.

\section{Results and Discussion}

First, it is important to consider what parameters may be important for the sensitivity of SCD ion sources. Clearly, when analyte ions are formed by chemical ionization, the ion residence time, $t_{\text {res, }}$ or the drift time of the reagent ions from the point of formation to the sampling orifice, will be important. Further, the total ion current $I$, passing through the orifice could conceivably be determined by the ion current density $I$, at the orifice wall,

$$
I=\int A
$$

where $A$ is the orifice surface area. Alternatively, $I$ could be determined by the ion density close to the orifice at the point where the ions become entrained in the viscous flow through the orifice, $\rho\left(t_{\text {res }}\right)$. In this case, $I$ is given by

$$
I=\rho\left(t_{\text {res }}\right) S
$$

where $S$ is the gas flow rate. This would seem to be a very reasonable assumption, and we will see later that eq 12 is in good agreement with experimental observations.

Here we will consider solutions to the space-charge. problem with particular emphasis on $\rho$ and $t_{\text {res }}$. Initially, the boundary value problem in the modeling of SCD ion sources and the analytical solution for the spherical geometry, eq 8 , is discussed. A more difficult geometry, the "needle-in-can," Figure 1e, is numerically approximated with a finite-element method. Finally, a simple geometry-independent treatment for ion transport and kinetics is presented and is used to calculate analytical sensitivities of a corona $\mathrm{API}$ ion source.

\section{Mathematical Modeling of Space-Charge- Dominated Ion Sources}

In most $\mathrm{SCD}$ ion sources, the primary ionization occurs in a (small) localized region. The remainder of the ion source constitutes the ion drift region, through which the ions are transported to the detector or vacuum orifice and where analytically useful ionmolecule reactions may occur. The modeling of the ionization region is difficult for a ${ }^{63} \mathrm{Ni}$ source and is very complicated for the corona API and likely also for the ESP source. Therefore, only the drift region will be modeled here. However, it will be shown that it is mainly in this region that the sensitivity of $\mathrm{SCD}$ ion sources is determined. As a consequence the treatment will be equally applicable to different ionization methods. It is also assumed that all ions have the same charge and the same mobility.

Excluding the ionization region in the modeling causes a problem with the boundary condition at the ion supply surface, eq 6 . Usually we know the total applied voltage, $V_{t}$,

$$
V_{t}=V_{i}+V_{d}
$$

where $V_{\mathrm{i}}$ is the voltage over the ionization region and $V_{\mathrm{d}}$ is the total ion drift voltage. Since we do not know $V_{i}$, there will be a range of possible potentials for the ion supply surface. This problem will be dealt with here by obtaining solutions for the whole range of physically reasonable boundary conditions.

Ions exit the drift region as they hit the ion source walls or pass through a vacuum orifice. The boundary condition here may be defined by specifying the potential or electric field at this surface.

It was mentioned in the Introduction that solutions to the space-charge problem are known for some 

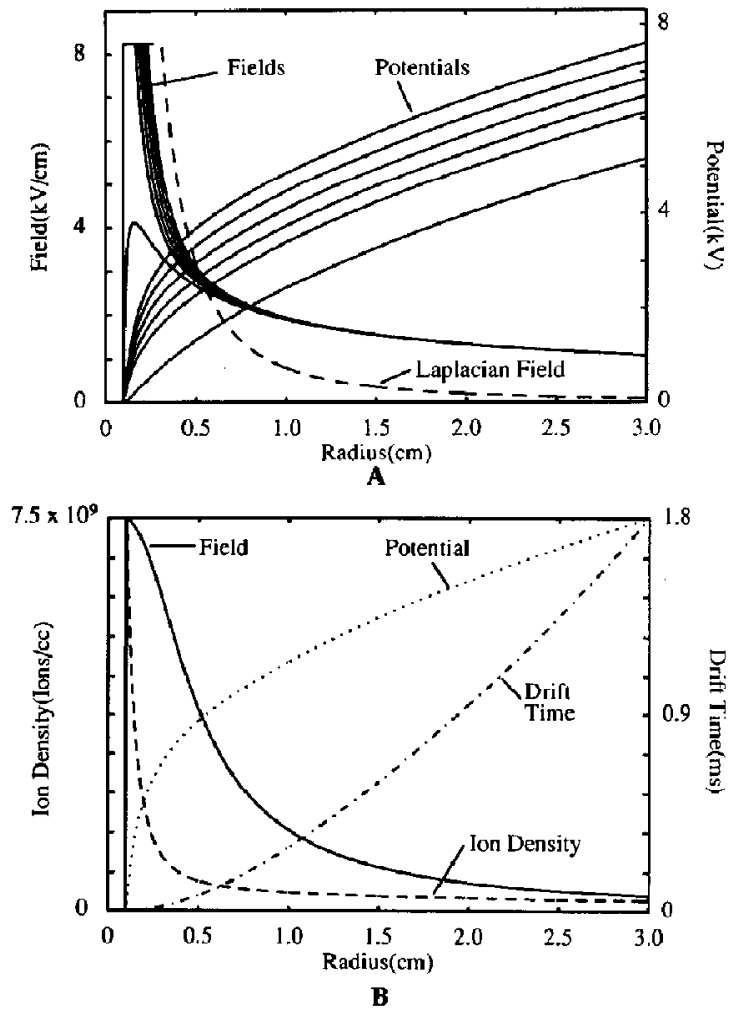

Figure 2. Space-charge effects in spherical geometry. Ion supply surface radius $0.1 \mathrm{~cm}$; outer electrode radius $3.0 \mathrm{~cm}$; discharge current $6 \mu \mathrm{A}$. (a) Field strengths and potential distributions versus radius for six values of applied potential $V_{d}$ as seen at radius $=3 \mathrm{~cm}$. At the space-charge limit, $V_{\mathrm{d}}=5224 \mathrm{~V}$ and $E_{0}=0 \mathrm{~V} / \mathrm{cm}$. Dashed line shows expected electric freld without space charge for $V_{d}=5224 \mathrm{~V}$. (b) Electric field, potential, ion density, and ion drift time for $V_{d}=7693 \mathrm{~V}$. Scales for drift time and ion density are shown. Full scale for electric field is 40,000 $\mathrm{V} / \mathrm{cm}$ and for potential $7693 \mathrm{~V}$.

simple geometries. It is instructive to first briefly discuss the spherical geometry, Figure 1c. Figure 2 shows calculated results for the spherical geometry with inner radius $r_{0}=0.1 \mathrm{~cm}$, outer radius $r_{1}=3 \mathrm{~cm}$, discharge current $I_{0}=6 \mu \mathrm{A}$, and mobility $\kappa=10^{-4}$ $\mathrm{m}^{2} /(\mathrm{V} \cdot \mathrm{s})$ typical for ions at atmospheric pressure [13]. The electric field was obtained from eq 8 , the potential by integration of eq 8 , the drift time $t_{\mathrm{d}}$ from eq 10 , and the ion density from eq 5 . Figure $2 \mathrm{a}$ shows the electric field and potential distributions for six different choices of $V_{\mathrm{d}}$ from 5224 to $7600 \mathrm{~V}$. The corresponding $E_{0}$ values range from 0 to $40 \mathrm{kV} / \mathrm{cm}$. At $1 \mathrm{~atm}$, substantial gas-phase ionization sets in at 30 $\mathrm{kV} / \mathrm{cm}$ [29], and for the drift region only fields lower than this would have to be considered. It is seen in Figure 2 that in the larger outer region of the ion source the electric field is insensitive to $E_{0}$ !

As the voltage $V_{d}$ is reduced toward $5224 \mathrm{~V}$, the electric field at the ion supply surface, $E_{0}$, rapidly decreases. In order to maintain a constant ion current,

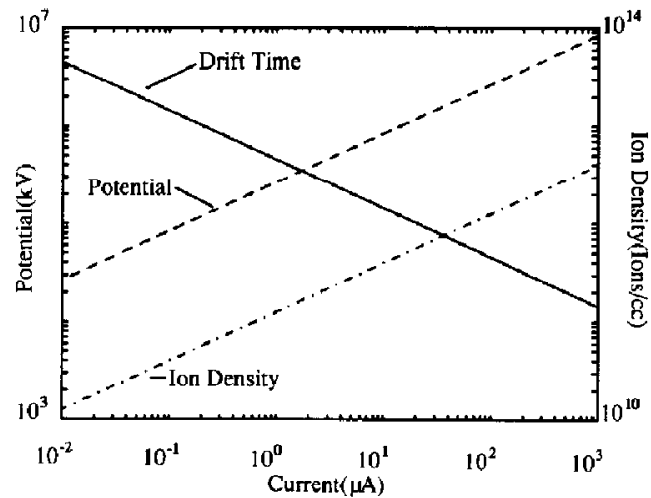

Figure 3. Variation of ion residence time, drift voltage, and ion density with discharge current; spherical ion source geometry as in Figure 3. The drift time scale is from $10^{-5}$ to $10^{-1} \mathrm{~s}$.

the ion density increases correspondingly. A further decrease in $V_{d}$ leads to an unphysical situation with a negative $E_{0}$ value. Therefore, $V_{0}=5224 \mathrm{~V}$ and $E_{0}=0$ $V / \mathrm{cm}$ represents the space-charge limit. Thus, $V_{\mathrm{d}}=$ $5224 \mathrm{~V}$ is the minimum voltage required to overcome the space charge in this particular case. Stated differently, at $V_{d}=5224 \mathrm{~V}$ the space-charge-limited (maximum) current [29] is $6 \mu \mathrm{A}$. This concept of a spacecharge limit is central to the understanding of SCD ion sources. The dashed line in Figure $2 \mathrm{a}$ shows the electric field distribution that $V_{\mathrm{d}}=5224 \mathrm{~V}$ would induce in the absence of space-charge effects-the Laplacian field. The difference relative to the SCD case is dramatic. With space charge, the electric fields are strongly suppressed close to the ion supply surface, whereas they are enhanced close to the counter electrode. Clearly, any analysis of these ion sources based upon Laplacian fields would likely be in error.

Figure $2 b$ shows the ion density, electric field, potential, and ion drift time as a function of radius for the special case of $V_{\mathrm{d}}=7693 \mathrm{~V}\left(E_{0}=40,000 \mathrm{~V} / \mathrm{cm}\right)$ and other parameters as in Figure 2a. Though not shown in the figure, the effect of changes in $V_{d}$ (or $E_{0}$ ) on ion drift time and ion density is very small. The decrease in ion density and the concurrent increase in ion drift time with radius are worth noting. That this behavior is fundamental to all SCD ion sources irrespective of geometry is shown later.

Of particular interest for practical applications is the dependence of the various source parameters on $I_{0}$ (total current) and on ion source size $r_{1}$. The dependence on $I_{0}$ is shown in Figure 3 with other parameters as in Figure 2. It is seen that $V_{\mathrm{d}}$ and $\rho_{\text {samp }}$ are proportional and $t_{\text {res }}$ inversely proportional to $I_{0}^{1 / 2}$. This also follows from eqs 14-16, which are easily derived for the space-charge limit and for $r_{0} \& r_{1}$.

$$
t_{\mathrm{res}} \approx\left(\frac{8 \pi \epsilon r_{1}^{3}}{3 \kappa I_{0}}\right)^{1 / 2}
$$




$$
\begin{gathered}
V_{\mathrm{d}} \approx\left(\frac{2 I_{0} r_{1}}{3 \pi \kappa \epsilon}\right)^{1 / 2} \\
\rho_{\text {samp }} \approx\left(\frac{3 \epsilon I_{0}}{8 \pi \kappa r_{1}^{3}}\right)^{1 / 2}
\end{gathered}
$$

Versions of eqs 15 and 16 can be found in refs. 21 and 22. The agreement between eqs 14-16 and the exact results in Figure 3 are excellent for $t_{\text {res }}$ and $\rho_{\text {samp }}$ whereas, as expected, there is a discrepancy for $V_{d}$. For a constant $l_{0}$, eqs 14-16 show that $t_{\text {res }}$ is propor tional and $\rho_{\text {samp }}$ inversely proportional to $r_{1}^{3 / 2}$, whereas $V_{\mathrm{d}}$ is proportional to $r_{1}^{1 / 2}$.

Equations 14-16 can be used to make order-ofmagnitude estimates of ion residence times, drift voltages, and ion sampling densities in any SCD ion source. Real ion sources, of course, do not have a spherical geometry but are usually much better approximated by the point-to-plane or "needle-in-can" geometry (Figure 1). Numerical solutions for such geometries [30], see also below, have shown that the space-charge-limited drift voltage for a point-to-plane geometry is approximately given by eq 15 , with $r_{1}$ being the point-to-plane distance, provided that the actual discharge current is multiplied by a factor of 2 .

The absolute sensitivity will behave as $\rho_{\text {samp }}$ if eq 12 is correct as anticipated. Thus, we would expect that in a corona discharge API or ESP ion source, the ion residence time, the ion sampling density, and thus the sensitivity are much more sensitive to moving the corona needle or ESP capillary in the source away from the sampling orifice than is the discharge voltage.

Because of its recent success [6], the extent to which the ESP ion source is space-charge-dominated is of particular interest. Because of the lateral extension of the spray, the geometry of this source would seem to be intermediate between the point-to-plane and planar geometries. The capillary-to-orifice distance is typically $3-5 \mathrm{~cm}$, and the applied voltage, $V_{t}$, is in the $1000-10,000 \mathrm{~V}$ range [31]. These values are very similar to corona API. However, the current in ESP is lower, $5 \times 10^{-8}$ to $10^{-6} \mathrm{~A}$ [31]. Using eq 15, with a correction factor of 2 for the current as mentioned above, we calculate $V_{\mathrm{d}}$ to be one-fourth to one-half of the total applied voltage as reported by Yamashita and Fenn [31]. However, in this calculation a mobility value of $1 \times 10^{-4} \mathrm{~m}^{2} /(\mathrm{V} \cdot \mathrm{s})$ was used, and most of the charge may be carried by droplets of unknown mobility. Even if the drift voltage $V_{\mathrm{d}}$ is a fraction of the total ESP voltage, it can be seen from Figure 2 that the electric field in the outer part of the ESP ion source is most certainly dominated by spacecharge effects.

In our treatment so far, we have assumed that all ions in the drift space have the same mobility. In ESP, the situation is much more complicated. The effect on the treatment presented here is solely through the mobility values. In ESP, the mobilities of ejected charged droplets are unknown. In addition, the droplets undergo largely unknown changes in radius and charge as they drift toward the orifice or capillary inlet. Finally, many of the formed ions are multiply charged, and again the mobility is unknown. To complicate matters further, it is not unlikely that the potential distribution caused by space charge of the droplets and the ions in turn influences the droplet evaporation and ion formation processes. Thus, the general conclusion that the ESP is space-chargedominated, with all the consequences that entails, is easy to make, but a detailed solution is likely very difficult. An interesting possibility is to use experimentally measured potential distributions in the ESP source to make deductions about the ionization process.

The point-to-plane geometry has been extensively studied for corona discharges. In Sigmond's model [22], it is assumed (1) that the (elliptical) Laplacian electric field lines are not affected by space charge and (2) that the electric field strength is constant along a field line from the point to the plane. Both turn out to be good approximations. Sigmond's model can be used to calculate both drift times and ion densities at the plane if the total voltage and current are known.

\section{Finite-Element Calculations for "Needle-in-Can" Geometry}

Because analytical solutions for the point-to-plane and needle-in-can geometries are not available, we have studied these using a funite-element method. A general description of the finite-element method for boundary value problems can be found in ref. 32 . Computational details related to the following discussion will appear elsewhere [30]. By substituting eq 4 with $F=0$ into eq 3 and substituting $-\rho / \epsilon$ for $\bar{\nabla} \cdot \bar{\nabla} U$ via eq 1 , one obtains a convection-diffusion equation,

$$
\kappa \bar{E} \cdot \bar{\nabla} \rho-D \bar{\nabla} \cdot \bar{\nabla} \rho=(\kappa / \epsilon) \rho^{2}
$$

To simulate a needle-in-can geometry, axial symmetry is assumed and the system of eqs 1 and 17 is converted to cylindrical coordinates on a rectangular computational domain $0<r<r_{1}, \quad r=\left(x^{2}+y^{2}\right)^{1 / 2}$. Boundary values for both $U$ and $\rho$ must be specified on all sides of the rectangle except along the $z$ axis, which corresponds to the axis of symmetry of the can. A uniform grid is set up on the rectangle, and a piecewise linear approximation is taken for both $U$ and $\rho$. A standard variational formulation of eqs 1 and 17 yields, respectively,

$$
\begin{gathered}
A u=b(\rho) / \epsilon \\
{[\kappa B(u)+D A]_{\rho}=(\kappa / \epsilon) c(\rho)}
\end{gathered}
$$

where $A$ and $B$ are matrices resulting from the discretization of the Laplacian operator and $\bar{E} \cdot \bar{\nabla}$, re- 
spectively. The components of the vectors $u$ and $\rho$ are, respectively, the approximate values of $U$ and $\rho$ at the grid points. The discrete nonlinear system of eqs 18,19 is then solved by using Newton's method.

Since typical values of $K E$ are much larger than the diffusion coefficient $D$ in eq 17 , a boundary layer forms at the "outflow boundary," where the electric field vector $E$ points out of the computational domain. This means that the density $\rho$ makes an extremely rapid transition from interior behavior to boundary behavior in a very narrow region near the outflow boundary. Failure to adequately resolve this boundary layer leads to (nonphysical) oscillations in the approximate solution throughout the computational domain. If a uniform mesh is used, the mesh spacing must be at least as small as the width of the boundary layer to prevent these oscillations. This leads to systems of equations with an intractably large number of unknown coefficients. To remedy this diffculty, we make use of a concept from computational fluid dynamics known as "artificial viscosity." Thus, the diffusion coefficient $D$ in eq 17 is made artificially large, about 1000 times larger than is typical at $1 \mathrm{~atm}$ [13]. This has the effect of producing a much wider boundary layer, which can be resolved with a much coarser grid with many fewer unknowns. The resulting approximation is poor near the outflow boundary but is quite accurate away from the boundary, where $\bar{\nabla} \boldsymbol{\rho}$ is small.

Figure 4 shows the result from finite-element calculations on an $11 \times 11$ grid for a needle-in-can geometry with $l=5 \mathrm{~cm}$ and $r_{1}=5 \mathrm{~cm}$ (Figure 1e). The ion supply surface, in front of the needle tip, has a radius of $1.0 \mathrm{~cm}$, a potential of $5 \mathrm{kV}$, and a charge density of $10^{-4} \mathrm{C} / \mathrm{m}^{3}\left(6.2 \times 10^{8}\right.$ ions $\left./ \mathrm{cm}^{3}\right)$. The charge density is zero at all other walls.

The only difference between Case 1 and Case 2 is in the boundary conditions. In Case 1, the potential of the can wall is $0 \mathrm{~V}$, whereas in Case 2 it is increased to $5 \mathrm{kV}$ in an effort to "focus" the ions down the center axis. The arrows in Figure $4 a$ and $b$ indicate the electric field. The divergence toward the can wall in Case 1 distinguishes this field from the nearly elliptical field in the point-to-plane geometry. The solid lines are equipotentials (in steps of $500 \mathrm{~V}$ ). Figure $4 \mathrm{c}$ and $\mathrm{d}$ show the charge density contours. The charge density is seen to decrease monotonically downfield from the ion supply surface. In Case 1 , the side wall of the can has a noticeable effect of "attracting" ions away from the center axis. The electric field strength along the axis at $r=0$ is shown in Figure $5 a$, and Figure $5 b$ shows the charge density along the same axis. Finally, Figure $5 c$ shows the current density distribution along the bottom of the can. It is interesting to note the radical difference in the axial field distribution, the much larger decrease in ion density for Case 2, and the rather moderate focusing of the ion current distribution achieved. It should be observed also that the total current is different in the two cases.
It is worth noting that the axial electric field for Case 1 (Figure $5 \mathrm{a}$ ) behaves similarly to that in the spherical case (Figure 2a) when close to the space-charge limit. Near the bottom of the can, however, the electric field again increases slightly. This is the qualitative behavior expected for the planar case, see eq 7 .

\section{Applications of the Unipolar Formula to SCD Ion Sources}

In the analytical and finite-element solutions above, it was seen that as the ions moved through the drift space the charge density decreased monotonically. That this should be a general phenomenon for SCD sources can be seen from the following argument. Imagine a small unipolar "cloud" of ions moving along the electric field lines through a gas. Each ion is drifting in the electric field induced by the charges and potentials in the surrounding space. For a small cloud, this field is the same for all ions. However, because the ions in the cloud also mutually repel each other, it follows that the cloud will expand. Mathematically, this expansion or space-charge "blow-out" [14] is expressed by the so-called unipolar charge drift formula [26]. Because of the importance of this formula, a short derivation will be given here.

The continuity equation, ignoring diffusion and gas convection, was given by eq 2 . In that equation, the derivative $\partial \rho / \partial t$ refers to a fixed point in space. The time derivative along the path of a charged cloud is given by

$$
\left(\frac{d \rho}{d t}\right)_{\text {path }}=\frac{\partial \rho}{\partial t}+\bar{W} \cdot \bar{\nabla} \rho
$$

where $\bar{W}$ is the ion drift velocity vector related to $\bar{J}$ and $o$,

$$
\bar{J}=\rho \bar{W}
$$

Using eqs 2, 20, and 1, we can write

$$
\begin{gathered}
\frac{\partial \rho}{\partial t}=-\bar{\nabla} \cdot \bar{J}=-\rho \bar{\nabla} \cdot \bar{W}-\bar{W} \bar{\nabla} \rho \\
\left(\frac{d \rho}{d t}\right)_{\text {path }}=-\rho \bar{\nabla} \cdot \bar{W}=-\rho \kappa \bar{\nabla} \cdot \bar{E}=\rho \kappa \bar{\nabla} \cdot \bar{\nabla} U \\
\left(\frac{d \rho}{d t}\right)_{\text {path }}=-\frac{\kappa \rho^{2}}{\epsilon}
\end{gathered}
$$

Integration of eq 24 from $t=0$ to $t=t$ yields

$$
\frac{1}{\rho(t)}=\frac{1}{\rho(t=0)}+\frac{\kappa}{\epsilon} t_{\mathrm{d}}
$$

This is the unipolar charge drift formula [22]. This is a very powerful formula for the analysis of $S C D$ ion sources. A main reason is that the formula is totally geometry-independent; it is equally valid for planar, 

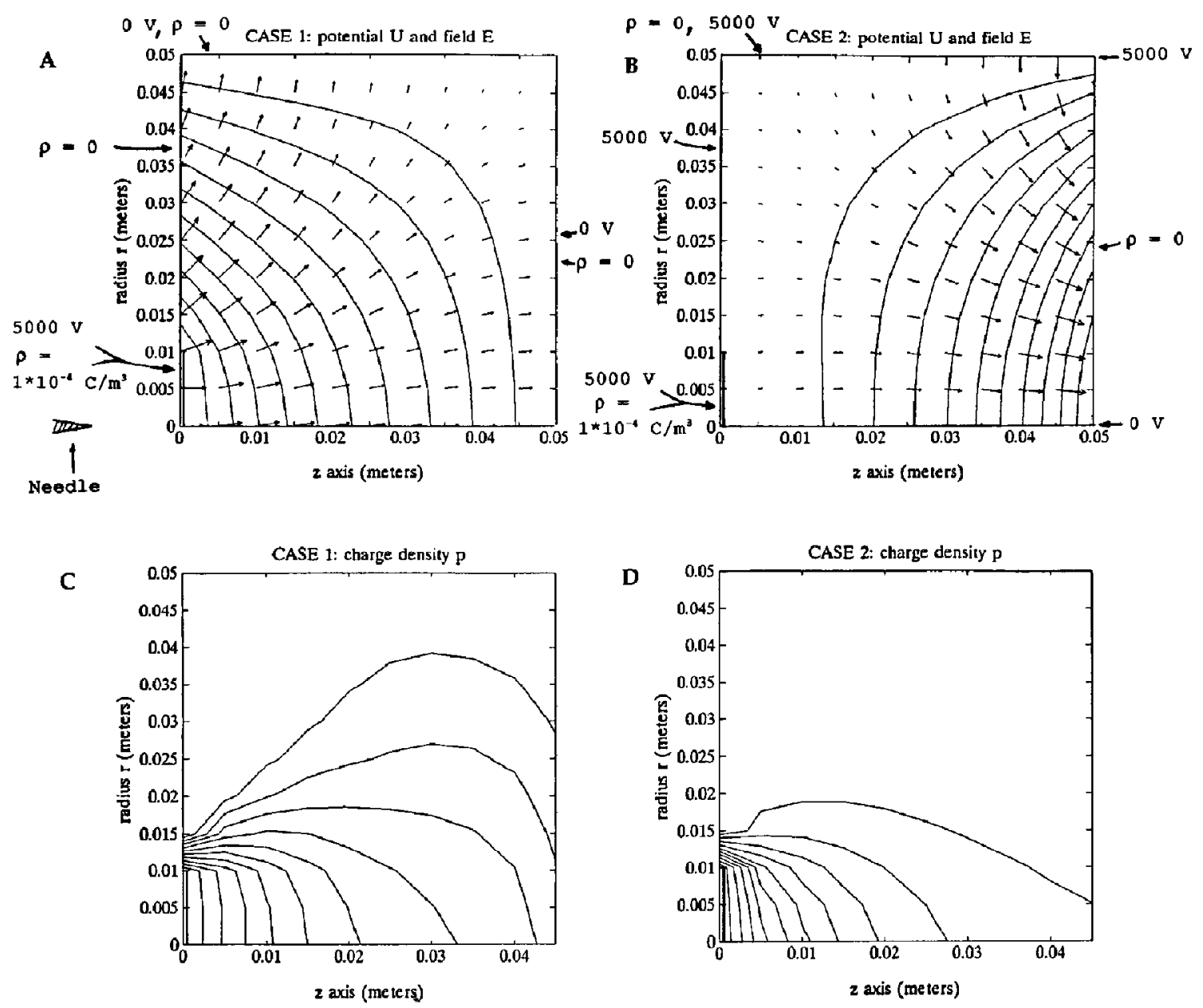

Figure 4. Results of finite-element calculations for the needle-in-can geometry. (a) Equipotential lines (500-V step) and electric field distribution and (c) ion density distribution $\left(10^{-5}-\mathrm{C} / \mathrm{m}^{3}\right.$ step) for Case 1. (b) and (d) show the same results for Case 2. Boundary conditions for Case 1: ion supply surface, $\rho=10^{-4} \mathrm{C} / \mathrm{m}^{3}$, in lower left corner; zero ion density at all other surfaces; can bottom, $z=0$, and wall, $r=5 \mathrm{~cm}$, at zero potential; ion supply surface at $5000 \mathrm{~V}$. Between the ion supply surface and the can wall, the potential decreases linearly. Case 2 differs from Case 1 in that the can wall and can top, $z=5 \mathrm{~cm}$, are at $5000 \mathrm{~V}$. Along the can bottom, the potential is proportional to $r^{2}$. The approximate position of a corona needle or ESP capillary is shown in (a).

cylindrical, spherical, and any other ion source geometry. However, the problem of calculating the residence time remains.

An interesting consequence of eq 25 is that in Laplacian fueld dominated ion sources the charge density will not change along the electric field lines. This may at first seem surprising. For example, in a spherical ion source, the ions obviously must spread out as they drift toward the outer electrode. However, the apparent conflict is resolved when it is realized that the ion cloud will compress in the radial direction as the electric field is decreasing.

Figure 6 shows ion densities calculated from the unipolar formula as a function of drift time for initial ion concentrations of (a) $10^{12}$, (b) $10^{11}$, and (c) $10^{10}$ ions $/ \mathrm{cm}^{3}$. A mobility value of $1 \times 10^{-4} \mathrm{~m}^{2} /(\mathrm{V} \cdot \mathrm{s})$ was used. It is seen that at long times the ion density is inversely proportional to ion drift time and independent of initial ion density. Thus, for a constant ion drift time, it is not possible to increase the ion signal by increasing the initial charge density as might be accomplished, for example, by increasing a discharge current.

An important consequence of the unipolar formula is that it is not possible to "concentrate" ions in an SCD ion source by applying any electric fields or by surrounding a low-charge-density region by high- 

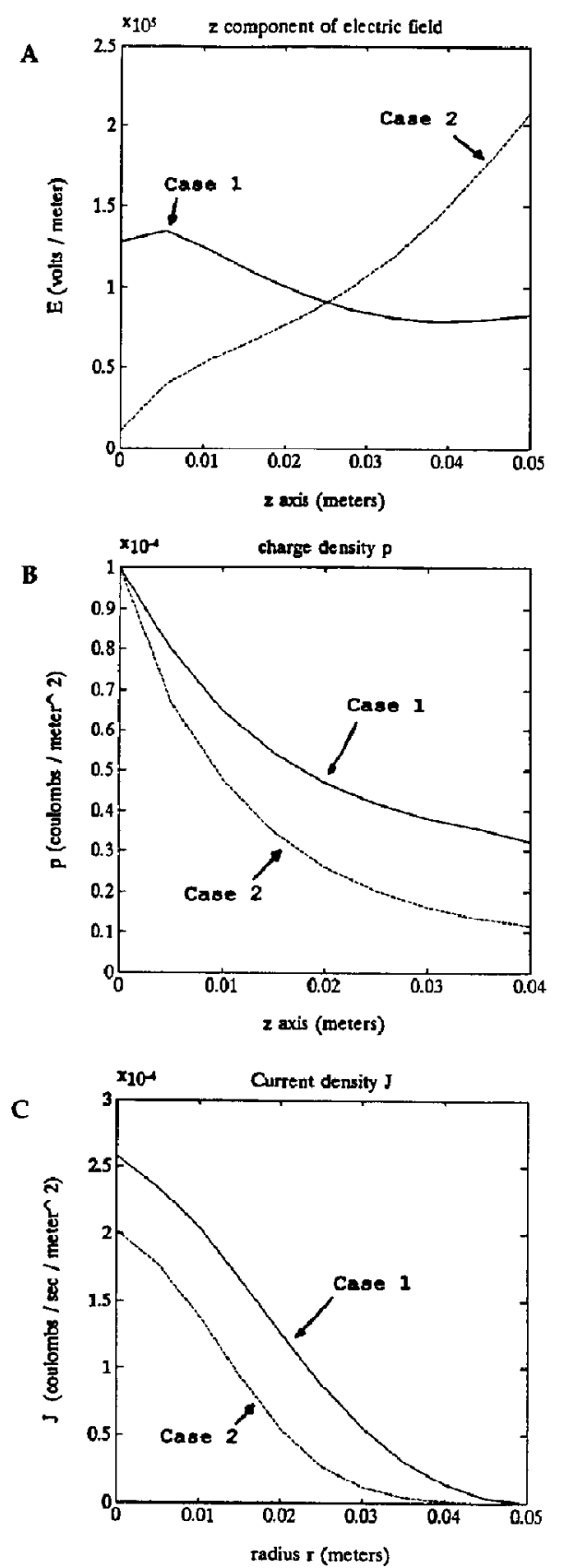

Figure 5. (a) Electric field and (b) ion density distribution along the $z$ axis $(r=0)$ and (c) ion current distribution along can bottom for Cases 1 and 2; see text and Figure 4 .

charge-density regions! Case 2 in Figures 4 and 5 illustrates this well. Comparing it with Case 1, it is seen that giving the can wall a high potential of $5000 \mathrm{~V}$ dramatically changes the electric field distribution and the ion current density, and charge density is somewhat concentrated toward the center axis of the source. However, it is seen in Figure $5 b$ that the

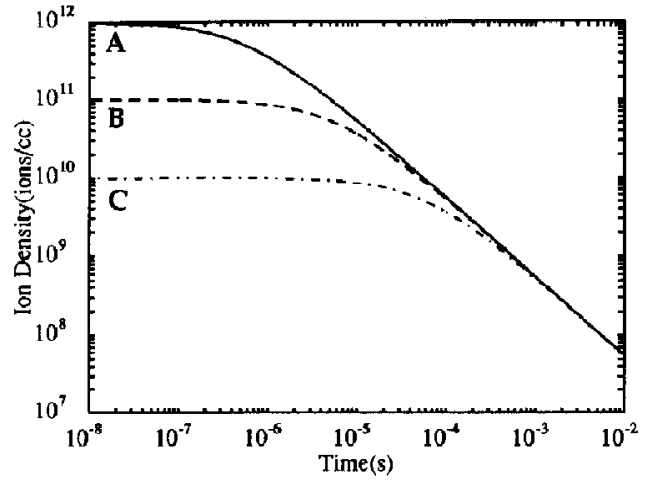

Figure 6. Ion density versus dritt time in a unipolar ion source for three values of $\rho_{0}$, the ton density at $t=0$, calculated from the ion drift formula, eq 21.

charge density at the can bottom, where the orifice is usually situated, is considerably lower in Case 2 than in Case 1. Thus, assuming that eq $\mathbf{1 2}$ is correct, the effort to concentrate the ions will result in a lower ion signal! The fact that such efforts have not been successful, as attested by the lack of such focusing in commercial instruments, also supports eq 12 over eq 11. For a constant distance and potential difference, the best strategy to maximize sensitivity is to keep a constant electric field throughout to the sampling orifice. This minimizes the ion residence time and, according to eq 25 , results in a maximum ion density at the orifice. This effect probably accounts for some moderate sensitivity effects observed experimentally [33]. However, it must also be remembered that we do not know the accuracy or limits of applicability of eq 12.

The unipolar charge drift formula will now be used to calculate absolute sensitivities in a corona API ion source for trace impurities. As the reagent ions, $R^{+}$, pass through the drift space, they ionize trace amounts of analyte, $P$,

$$
\mathrm{R}^{+}+\mathrm{P}^{k_{26}} \rightarrow \mathrm{P}^{+}+\mathrm{R}
$$

where $k_{26}$ is the (second-order) rate constant. It is assumed that the reverse reaction does not occur, that is, we are dealing with an analyte whose sensitivity is kinetically as opposed to thermodynamically controlled [27]. In ambient air, the reagent ions are $\mathrm{H}_{3} \mathrm{O}^{+}\left(\mathrm{H}_{2} \mathrm{O}\right)_{h}$,

$\mathrm{H}_{3} \mathrm{O}^{+}\left(\mathrm{H}_{2} \mathrm{O}\right)_{h}+\mathrm{B} \rightarrow \mathrm{BH}^{+}\left(\mathrm{H}_{2} \mathrm{O}\right)_{b}+(h-b+1) \mathrm{H}_{2} \mathrm{O}$

From eq 12, the current of product ions through the orifice is given by

$$
I_{\mathrm{P}^{+}}=\rho\left(t_{\mathrm{res}}\right) S\left[1-\exp \left(-k_{22} N_{\mathrm{P}} t_{\text {res }}\right)\right]
$$

where $N_{P}$ is the number density of analyte molecules.

For an analyte whose sensitivity is thermodynami- 


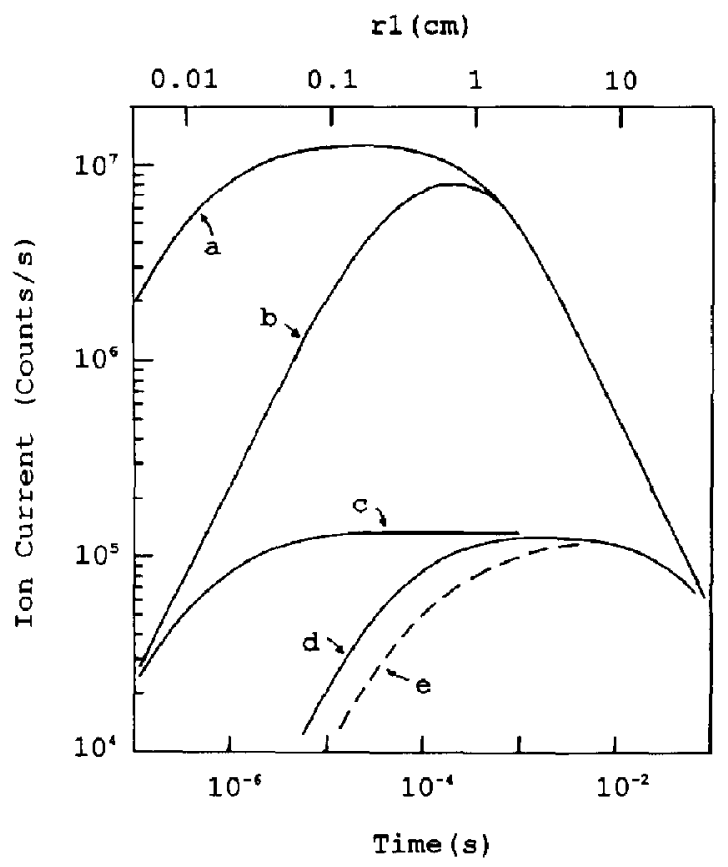

Figure 7. Analyte ion intensities in a corona API source versus ion drift time calculated with eq 25 . The upper $x$ axis shows needle-to-orifice distance for a discharge current of $3 \mu \mathrm{A}$; see text. (a) $\rho_{0}=10^{12}$ ions $/ \mathrm{cm}^{3}, c_{\mathrm{a}}=100 \mathrm{ppb}$; (b) $\rho_{0}=10^{10}$ ions $/ \mathrm{cm}^{3}, c_{\mathrm{a}}=100 \mathrm{ppb}$; (c) $\rho_{0}=10^{12}$ ions $/ \mathrm{cm}^{3}, c_{\mathrm{a}}=1 \mathrm{ppb}$; (d) $p_{0}=10^{10}$ ions $/ \mathrm{cm}^{3}, c_{\mathrm{a}}=1 \mathrm{ppb}$; (c) as (c) but $4-\mathrm{mm}$ curtain gas in front of orifice.

cally controlled [27], eq 25 should be substituted simply by

$$
I_{\mathrm{P}^{+}}=\rho\left(t_{\mathrm{res}}\right) \mathrm{S} \frac{\left[\mathrm{P}^{+}\right]}{\sum\left[\mathrm{H}_{3} \mathrm{O}^{+}\left(\mathrm{H}_{2} \mathrm{O}\right)_{h}\right]+\left[\mathrm{P}^{+}\right]}
$$

where $\left[\mathrm{P}^{+}\right] /\left(\Sigma\left[\mathrm{H}_{3} \mathrm{O}^{+}\left(\mathrm{H}_{2} \mathrm{O}\right)_{h}\right]+\left[\mathrm{P}^{+}\right]\right.$is the equilibrium ion concentration ratio.

The calculation of the sensitivity of a typical corona API source by use of eq 28 will now be illustrated. The following conditions are assumed. The geometry is point-to-plane with $I_{0}=3 \mu \mathrm{A}$. The rate constant for formation of analyte ions is $k_{26}=1 \times 10^{-9}$ $\mathrm{cm}^{3} /($ molecule $\cdot \mathrm{s})$. The gas flow through the $0.1-\mathrm{mm}$ ion-sampling orifice is calculated to be about $1.0 \mathrm{~cm}^{3} / \mathrm{s}$ at $1 \mathrm{~atm}$ [34]. The fraction of ions entering the orifice that reaches the mass spectrometer detector was estimated to be $1 \%$. This represents a reasonable guess only. For these conditions, Figure 7 shows calculated analyte ion signals as a function of ion residence time for three different combinations of analyte concentrations and initial (reagent) ion density. At short $t_{\text {tes }}$ the analyte ion signal increases with $t_{\text {res }}$. The reason is that conversion from reagent to analyte ions increases while $\rho\left(t_{\text {res }}\right)$ remains relatively constant; see the initial flat portion of the graphs in Figure 6. At long $t_{\text {res }}$, on the other hand, the conversion to analyte

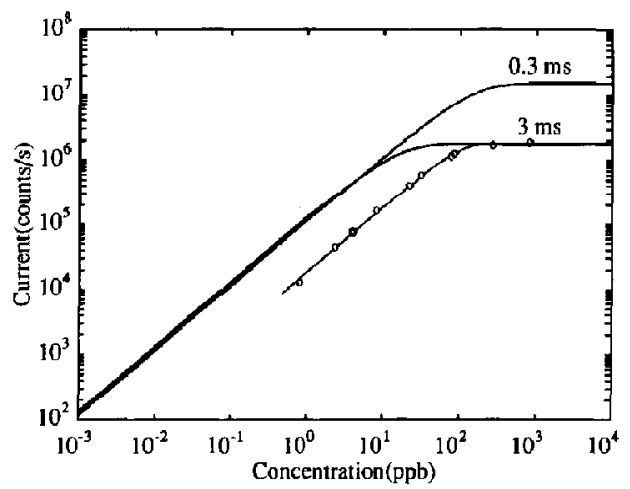

Figure 8. Analyte ion signal versus analyte concentration in an API ion source for an analyte with kinetically controlled sensitivity. Upper lines show calculated results for ion residence times of 0.3 and $3 \mathrm{~ms}$, respectively; experimental points from ref. 27 .

ions is nearly complete, and the ion signal decreases as $p\left(t_{\text {res }}\right)$ decreases. The most noteworthy feature in Figure 7 is the intermediate plateau region for lowconcentration analytes, where the analyte ion signal does not change appreciably. The reason for this is that the increase in $t_{\text {res }}$ is nearly canceled by a corresponding decrease in $\rho\left(t_{\mathrm{res}}\right)$. The plateau was experimentally observed in the author's previous article (see ref 27$)$. We observe that with eq 11 such a plateau would not be predicted; instead $l_{\mathrm{P}}+$ would be proportional to $r_{1}^{-(1 / 2)}$. The upper axis in Figure 7 shows the needle-to-orifice distance calculated from the ion drift time and eq 13 with $l_{0}=3 \mu \mathrm{A}$. It is seen that in order to obtain a drift time of less than $10^{-6} \mathrm{~s}$, the needle-to-orifice distance must be made very small and comparable to the orifice radius. Because the ionization processes as well as the initial ion-molecule chemistry become very important at short distances, and also because one can expect severe disturbances in the ion-sampling mechanism, a more detailed model would be required for these short times.

In some instruments, a "curtain gas," for example dry $\mathrm{N}_{2}$, is used in front of the orifice. In the curtain gas, the unipolar formula still holds, but reactions 26 or 27 are quenched. A comparison of curves $c$ (no
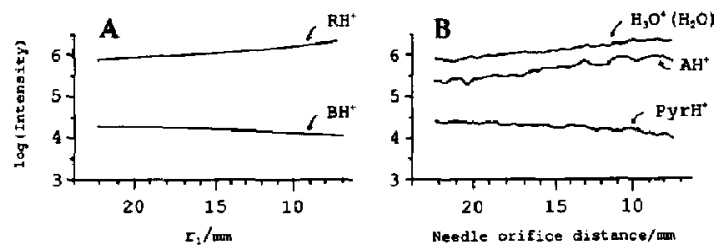

Figure 9. Reagent and analyte (pyridine) ion signals versus needle-to-orifice distance in an API ion source with $0.1 \mathrm{ppb}$ pyridine. (a) Calculated results, source conditions as in Figure 6e; $\mathrm{RH}^{+}=$reagent ion; $\mathrm{BH}^{+}=$analyte ion. (b) Experimental results from ref. $27 . \mathrm{AH}^{+}$is protonated acetone, the intensity of which is thermodynamically controlled. 
curtain gas) and e (4 mm of curtain gas) in Figure 7 illustrates the effect of the curtain gas. The ion residence time in the curtain gas was calculated by using eq 14 , and it varied in the $10^{-4}-10^{-3}$ s range. $A$ residence time in the curtain gas of $0.2 \mathrm{~ms}$ was estimated previously from experimental data [27].

Figure 8 shows a calculation of analyte ion currents as a function of concentration for two different residence times. The same source conditions as for Figure 7 were used. The circles show corresponding experimental results [27]. The agreement is good considering that the ion intensities were artificially suppressed by about a factor of 10 in the experiments. The residence time was estimated to be $0.3 \mathrm{~ms}$ [27], also in good agreement with the present calculations (Figure 8).

Finally, Figure 9a shows calculated intensities for $0.1 \mathrm{ppb}$ of pyridine in ambient air with $k_{26}=2 \times 10^{-9}$ $\mathrm{cm}^{3} /($ molecule $\cdot \mathrm{s}$ ) as a function of the distance from the needle to the curtain gas orifice. The corresponding experimental result is shown in Figure 9b [27]. The introduction of 4-mm-deep curtain gas into the calculations has the effect of slightly curving the analyte ion signal downwards with decreasing needle-toorifice distance, in agreement with experiment.

\section{Acknowledgment}

Mike Ikonomou is thanked for helping J. S. in a doomed effort to improve the sensitivity of API/MS. This work was partly supported by a grant from the Montana/EPSCOR II Program of the National Science Foundation (grant no. R11-8921978).

\section{References}

1. French, J. B.; Thomson, B. A.; Davidson, W. R.; Reid, N. M.; Buckley, J. A. In Mass Spectrometry in the Environmental Sciences; Karasek, F. W.; Hutzinger, O.; Safe, S., Eds.; Plenum: New York, 1984; pp 101-121.

2. Mitchum, R. K.; Korfmacher, W. A. Anal. Chem. 1983, 55, 1485A.

3. Proctor, C. J.; Todd, J. F. J. Org. Mass Spectrom. 1983, 18, 509.

4. Ketkar, S. N.; Dulak, J. C.; Fite, W. L.; Buchner, J. D.; Dheandhanoo, S. Anal. Chem. 1989, 61, 260.
5. Covey, T.; Lee, E.; Bruins, A.; Henion, J. Anal. Chem. 1986, $58,1451 \mathrm{~A}$.

6. Fenn, J. B.; Mann, M.; Meng, C. K.; Wong, S. F.; Whitehouse, C. M. Science 1989, 246, 64.

7. Wong, S. F.; Meng, C. K.; Fenn, J. B. J. Phys. Chem. 1988, 92,546 .

8. Lovelock, J. E, Anal. Chem. 1963, 35, 474.

9. Dressler, M. Selective Gas Chromatographic Detectors; Elsevier: Amsterdam, 1986; p 217.

10. Mock, R. S.; Grimsrud, E. P. Anal. Chem. 1988, 60, 1684.

11. Dam, R. In Plasma Chromatography; Carr, T. W., Ed.; Plenum: New York, 1984; pp 177-213.

12. Kevercomb, H. E.; Mason, E. A, Anal. Chem. 1975 47, 970.

13. McDaniel, E. W.; Mason, E. A. The Mobility and Diffusion of Ions in Gases; Wiley: New York, 1973.

14. Gobby, P. L.; Grimsrud, E. P.; Warden, S. W. Antal. Chem. 1980, 52, 473.

15. Carroll, D. I.; Dzidic, I; Stillwell, R. N.; Horning, M. G.; Horning, E. C. Anal. Chem. 1974, 46, 706.

16. Siegel, M. W.; McKeown, M. C. J. Chromatogr. 1976, 122, 397.

17. Siegel, M. W. In Plasma Chromatography; Carr, T., Ed.; Plenum: New York, 1984; pp 95-113.

18. Loeb, L. B. Electrical Coronas. Their Basic Physical Mechanisms; University of California Press: Berkeley, 1965.

19. Tuwnsend, J. S. Phil. Mag. 1914, 28, 83.

20. Langmuir, I. Phys. Rev. 1913, 2, 450.

21. Chapman, S. J. Atmos. Sci. 1977, 34, 1801.

22. Sigmond, R. S. In Electrical Breakdown of Gases; Meek, J. M.; Craggs, J. D., Eds., Wiley: Chichester, 1978; pp 319-384.

23. Hinds, W. C. Aerosol Technology. Properties, Behavior, and Measurement of Airborne Particles; Wiley: New York, 1982.

24. Rubinstein, I. Electro-diffusion; SIAM: Philadelphia, 1990.

25. Selberherr, S. Analysis and Simulation of Semiconductor Devices; Springer-Verlag: Wien, 1982.

26. Sigmond, R. S. T. Appl. Phys. 1982, 53, 891.

27. Sunner, J.; Nicol, G; Kebarle, P. Anal. Chem. 1988, 60, 1300.

28. Sunner, J.; Ikonomou, M.; Kebarle, P. Anal. Chem. 1988, $60,1308$.

29. Loeb, L. B. Basic Processes of Gaseous Electronics; University of California Press: Berkeley, 1955.

30. Vogel, C.: Busman, M.; Sunner, J. in preparation.

31. Yamashita, M.; Fenn, J. B. J. Phys. Chem. 1984, 88, 4451.

32. Axelsson, O.; Barker, V. A. Finite Element Solutions to Boundary Value Problems; Academic: New York, 1984.

33. Potjewyd, J. Focussing of Ions in Atmospheric Pressure Gases Using Electrostatic Fields, Ph.D, thesis, University of Toronto, 1983.

34. Dushman, S. Scientific Fourldulions of Vucuum Technique; Wiley: New York, 1958. 